this and other studies does not contraindicate a diagnosis of migraine. Migraine-epilepsy syndromes are reviewed by Andermann F. Boston: Butterworths $1987 ; 407-8$. These include epileptic seizures induced by a classic migraine aura and seen more often in children than in adults.

\title{
MIGRAINE AND EPILEPSY: INFANTILE ONSET
}

The coexistence of classical migraine, visual phenomena and seizures and occipital spike-wave complexes in the EEG are reported in 14 children from the Department of Neurological Sciences, University La Sapienza, Rome, Italy. The age of onset was 3 to 9 years (mean age 5.4 years) and the clinical and EEG follow-up ranged from 4 to 16 years (mean 11.2 years). Visual phenomena were described as bright rings, bands, red-colored disks, in both visual fields or in the visual hemifield opposite the spike-wave localization. Amaurosis followed the appearance of the phosphenes. In some patients the migraine with aura and epilepsy were chronologically related phenomena; migraine might occur immediately before or after seizures; migraine may act as a triggering factor for epilepsy. In others, visual phenomena either preceded migraine with aura or occurred as an isolated event or even preceded epileptic seizures (De Romanis F et al. Migraine and epilepsy with infantile onset and electroencephalographic findings of occipital spike-wave complexes.

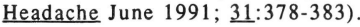

COMMENT. In $50 \%$ of the patients in this study, migraine with aura, with phosphenes and amaurosis as prodromal symptoms, replaces epileptic seizures when they disappear or improve following antiepileptic treatment. Ergot alkaloids or pizotifen were of little or no benefit whereas migraine with aura responded to anti-epileptic treatment. Occipital epilepsy in childhood in this study was not benign and seizures were controlled by anti-epileptic treatment in only 2 subjects.

Migraine and pregnancy is reviewed by Uknis A and Silberstein SD in the same issue of Headache 1991; 31:372-374. A first attack of migraine with unilateral parasthesias and blurred vision occurred in a 27-year-old woman during the first trimester and led to the diagnosis of her pregnancy. Pre-existing migraine usually improves with pregnancy, whereas relapse frequently occurs in the post-partum period. Children born to migraineurs have no increased incidence of birth defects.

\section{SEIZURE DISORDERS}

\section{PROGNOSIS OF NEONATAL SEIZURES}

The neurologic outcome of 40 infants with EEG documented seizures of diverse etiologies was examined retrospectively at the Division of Neurology, The Children's Hospital of Philadelphia, PA. Neurologic followup of 27 survivors performed at a mean age of 31 months (range 5 to 56 
months) was favorable in $30 \%$ of the study population. The remaining $70 \%$ (28 of 40 ) with an unfavorable outcome included mortality in $33 \%$ and severe morbidity in $38 \%$. Epilepsy occurred in $56 \%$ of survivors (15 of 27), development delay - $67 \%$, and cerebral palsy - $63 \%$. Factors influencing the outcome were the etiology of the seizures, the age at the onset of seizures, birth weight and neurologic examination results. Asphyxia, meningitis and cerebral dysgenesis carried a poor prognosis. Onset of seizures within the first day of life and a severely abnormal neurologic exam significantly predicted an unfavorable outcome. An Apgar score less than 5 at 5 minutes was not significantly related to a poor prognosis. The EEG background activity was the only EEG parameter of value in prognosis: an abnormal background carried an unfavorable outcome in $81 \%$ and a normal background was predictive of a favorable prognosis in $67 \%$. (Legido A et al. Neurologic outcome after electroencephalographically proven neonatal seizures.

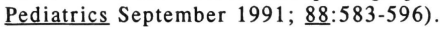

COMMENT. In this retrospective study of neonates with EEG proven seizures, the mortality rate was $33 \%$ and the morbidity rate was $56 \%$.

The long-term outcome of an infant with pyridoxine-dependent neonatal seizures is reported from the British Columbia's Children's Hospital, Vancouver, BC, Canada (Connolly MB et al. Intravenous immunoglobulin and pyridoxine-dependent seizures. Neurology September $1991 ; 41: 1524)$. Generalized clonic seizures began at 6 days of age followed by partial motor seizures becoming secondarily generalized at 1 month, and episodes of status epilepticus lasting 1 hour at $2-1 / 2$ months. Seizures were controlled and the EEG normalized by $100 \mathrm{mg}$ pyridoxine daily. The patient remained seizure free until age 20 months and then developed episodes of status epilepticus twice a week, always associated with infections. Monthly IV gamma globulin therapy $400 \mathrm{mg} / \mathrm{kg}$ infused over 6 hours resulted in prevention of infections and control of convulsions. IVIg therapy may be helpful in the treatment of pyridoxine-dependent seizures beginning in the neonatal period and may improve the long-term outcome.

\section{SURGERY IN WEST SYNDROME}

The results of surgical removal of a porencephalic cyst in an 18-month old child with infantile spasms and hypsarrhythmia are reported from the Departments of Neurology and Neurosurgery, VA Medical Center and University of Florida; the State University of Stony Brook, NY; and University of Michigan Medical School, Ann Arbor, MI. Infantile spasms were evaluated first at 4 months of age. The seizures were generalized but the neurologic examination showed a left hemiparesis and a left visual field deficit. ACTH provided a transient control of spasms for 3 months. At 18 months the seizures had become refractory to medical treatment. Postoperatively, seizures have been controlled for 12 months and the use of the paretic extremities has improved. Preoperative anti-epileptic medications 